\title{
Bounds on negative energy densities in flat spacetime
}

\author{
C.J. Fewster* and S.P. Eveson ${ }^{\dagger}$ \\ Department of Mathematics, University of York, \\ Heslington, York YO1 5DD, United Kingdom
}

(May 7, 1998)

\begin{abstract}
We generalise results of Ford and Roman which place lower bounds - known as quantum inequalities - on the renormalised energy density of a quantum field averaged against a choice of sampling function. Ford and Roman derived their results for a specific non-compactly supported sampling function; here we use a different argument to obtain quantum inequalities for a class of smooth, even and non-negative sampling functions which are either compactly supported or decay rapidly at infinity. Our results hold in $d$-dimensional Minkowski space $(d \geq 2)$ for the free real scalar field of mass $m \geq 0$. We discuss various features of our bounds in 2 and 4 dimensions. In particular, for massless field theory in 2-dimensional Minkowski space, we show that our quantum inequality is weaker than Flanagan's optimal bound by a factor of $\frac{3}{2}$.
\end{abstract}

*Electronic address: cjf3@york.ac.uk

$\dagger$ †lectronic address: spe1@york.ac.uk 


\section{INTRODUCTION}

The stress-energy tensor $T_{a b}$ is said to obey the weak energy condition (WEC) if $T_{a b} u^{a} u^{b} \geq 0$ for all timelike vectors $u^{a}$. This condition is obeyed by all known forms of classical matter, but is violated in quantum field theory [1] in which the renormalised energy density may become arbitrarily negative at points of spacetime. If extended regions of large negative energy density occur in nature, a variety of exotic phenomena might be possible, ranging from violations of the second law of thermodynamics and cosmic censorship to the creation of time machines and 'warp drive'. Accordingly, it is important to understand the extent to which the weak energy condition may be violated. In a series of papers [2 [6], Ford, Roman and Pfenning have studied quantum field theory in various flat and curved spacetimes and established lower bounds (known as quantum inequalities) on the time averaged energy densities measured by observers.1 The results have been employed to argue against the possibility of traversable wormholes [8], warp drive [9] and also to discuss the rôle of negative energy densities in the process of black hole evaporation [10].

To be specific, consider a free real scalar field of mass $m$ in $d$-dimensional Minkowski space, and let $T_{a b}^{\mathrm{ren}}$ denote the renormalised stress-energy tensor. Quantum inequalities provide lower bounds on the averaged expected energy density

$$
\rho_{f, \psi}=\int_{-\infty}^{\infty} d t\left\langle T_{00}^{\mathrm{ren}}(t, \mathbf{0})\right\rangle_{\psi} f(t)
$$

measured by a stationary observer at the spatial origin $\mathbf{0}$, where $f$ is a non-negative sampling function and the angle brackets denote the expectation value in the quantum state $\psi$. The sampling function employed by Ford et al. is the Lorentzian function peaked at $t=0$

$$
L(t)=\frac{\tau}{\pi\left(t^{2}+\tau^{2}\right)}
$$

in which $\tau$ sets the timescale over which sampling occurs. With this sampling function, Ford and Roman have shown [3] that in four dimensional Minkowski space the averaged energy density $\rho_{f, \psi}$ obeys the bound

$$
\rho_{L, \psi} \geq-\frac{3}{32 \pi^{2} \tau^{4}} G(2 m \tau)
$$

for all (sufficiently well-behaved) states $\psi$ and any $\tau>0$, where the real-valued function $G$ is independent of $\psi$ and is positive and strictly decreasing on $\mathbb{R}^{+}$with $G(0)=1$. In two dimensions, they obtained the corresponding result

$$
\rho_{L, \psi} \geq-\frac{1}{8 \pi \tau^{2}} F(2 m \tau)
$$

where $F(y)=\frac{1}{2} y^{2}\left(K_{0}(y)+K_{2}(y)\right)$ and the $K_{\nu}$ are modified Bessel functions of the second kind.

\footnotetext{
${ }^{1}$ Interestingly, it turns out that space averaged energy densities in 4-dimensions are not bounded below [7].
} 
In the present paper, we will improve and generalise these bounds to cover more general sampling functions in Minkowksi space of arbitrary dimension. Some progress in this direction has already been made by Flanagan [11] in the case of 2-dimensional massless field theory. Flanagan derived an optimal lower bound on $\rho_{f, \psi}$ given by

$$
\min _{\psi} \rho_{f, \psi}=-\frac{1}{24 \pi} \int_{\operatorname{supp} f} d t \frac{\left(f^{\prime}(t)\right)^{2}}{f(t)}
$$

for any smooth non-negative sampling function $f$, where $\operatorname{supp} f$ denotes the support of $f$. F Flanagan's argument depends critically on special features of 2-dimensional massless field theory. In this paper, we will consider arbitrary smooth, non-negative, even sampling functions $f$ of rapid decrease at infinity (including the possibility of compact support), and will obtain the slightly weaker bound

$$
\rho_{f, \psi} \geq-\frac{1}{16 \pi} \int_{\operatorname{supp} f} d t \frac{\left(f^{\prime}(t)\right)^{2}}{f(t)}
$$

in the 2-dimensional massless case. However, our argument has the virtue of generalising directly to both massive field theory in two dimensions and to massive and massless field theory in four dimensional Minkowski space. Our general quantum inequality in $n+1$ dimensional Minkowski space, is the bound

$$
\rho_{f, \psi} \geq-\frac{C_{n}}{2 \pi(n+1)} \int_{m}^{\infty} d u\left(\widehat{f^{1 / 2}}(u)\right)^{2} u^{n+1} Q_{n}\left(\frac{u}{m}\right),
$$

for all (sufficiently well-behaved) quantum states $\psi$, where $f^{1 / 2}(t)=\sqrt{f(t)}$, the hat denotes the Fourier transform and the constant $C_{n}$ is equal to the area of the unit $n-1$-sphere divided by $(2 \pi)^{n}$. The bounded non-negative functions $Q_{n}$ are defined (for $n \geq 1$ ) on $[1, \infty$ ) by

$$
Q_{n}(x)=(n+1) x^{-(n+1)} \int_{1}^{x} d y y^{2}\left(y^{2}-1\right)^{n / 2-1},
$$

and obey $Q_{n}(1)=0$ and $Q_{n}(x) \rightarrow 1$ as $x \rightarrow \infty$. We plot $Q_{1}$ and $Q_{3}$ in Figures 1 and 2 .

The derivation of Eq. (1.7) is quite simple and depends mainly on the canonical commutation relations and the convolution theorem. Although we will argue formally here, we expect that the elementary nature of the argument will facilitate a fully rigorous treatment.

\section{PRELIMINARIES}

We begin by stating our conventions. In $n+1$-dimensional Minkowski space, $\eta_{a b}$ denotes the metric with signature $+-\cdots-$. The Klein-Gordon equation is

$$
\left(\square+m^{2}\right) \varphi \equiv\left(\eta^{a b} \partial_{a} \partial_{b}+m^{2}\right) \varphi=0
$$

\footnotetext{
${ }^{2}$ Flanagan also derives related quantum inequalities for other components of $T_{a b}^{\mathrm{ren}}$.
} 
and the quantum field $\Phi(x)$ is defined by

$$
\Phi(x)=\int \frac{d^{n} \mathbf{k}}{(2 \pi)^{n} \sqrt{2 \omega(\mathbf{k})}}\left(a(\mathbf{k}) e^{-i k \cdot x}+a^{\dagger}(\mathbf{k}) e^{i k \cdot x}\right)
$$

where $\omega(\mathbf{k})=\sqrt{\|\mathbf{k}\|^{2}+m^{2}}$, the $n+1$-vector $k^{a}$ has components $(\omega(\mathbf{k}), \mathbf{k})$, and the annihilation and creation operators $a(\mathbf{k})$ and $a^{\dagger}(\mathbf{k})$ obey the canonical commutation relations

$$
\left[a(\mathbf{k}), a\left(\mathbf{k}^{\prime}\right)\right]=\left[a^{\dagger}(\mathbf{k}), a^{\dagger}\left(\mathbf{k}^{\prime}\right)\right]=0 \quad\left[a(\mathbf{k}), a^{\dagger}\left(\mathbf{k}^{\prime}\right)\right]=(2 \pi)^{n} \delta\left(\mathbf{k}-\mathbf{k}^{\prime}\right) .
$$

The classical energy density of a field $\varphi$ is

$$
T_{00}=\frac{1}{2}\left\{\left(\partial_{0} \varphi\right)^{2}+\sum_{i=1}^{n}\left(\partial_{i} \varphi\right)^{2}+m^{2} \phi^{2}\right\},
$$

from which the renormalised (normal ordered) quantum energy density at position $(t, \mathbf{0})$ is easily shown to be

$$
\begin{aligned}
T_{00}^{\mathrm{ren}}(t, \mathbf{0})= & \int \frac{d^{n} \mathbf{k} d^{n} \mathbf{k}^{\prime}}{(2 \pi)^{2 n}}\left[\frac{\omega(\mathbf{k}) \omega\left(\mathbf{k}^{\prime}\right)+\mathbf{k} \cdot \mathbf{k}^{\prime}}{4\left(\omega(\mathbf{k}) \omega\left(\mathbf{k}^{\prime}\right)\right)^{1 / 2}}\left\{a^{\dagger}(\mathbf{k}) a\left(\mathbf{k}^{\prime}\right) e^{i\left(\omega(\mathbf{k})-\omega\left(\mathbf{k}^{\prime}\right)\right) t}-a(\mathbf{k}) a\left(\mathbf{k}^{\prime}\right) e^{-i\left(\omega(\mathbf{k})+\omega\left(\mathbf{k}^{\prime}\right)\right) t}\right\}\right. \\
& \left.+\frac{m^{2}}{4\left(\omega(\mathbf{k}) \omega\left(\mathbf{k}^{\prime}\right)\right)^{1 / 2}}\left\{a^{\dagger}(\mathbf{k}) a\left(\mathbf{k}^{\prime}\right) e^{i\left(\omega(\mathbf{k})-\omega\left(\mathbf{k}^{\prime}\right)\right) t}+a(\mathbf{k}) a\left(\mathbf{k}^{\prime}\right) e^{-i\left(\omega(\mathbf{k})+\omega\left(\mathbf{k}^{\prime}\right)\right) t}\right\}+\text { h.c. }\right] .
\end{aligned}
$$

Finally, the Fourier transform $\widehat{f}$ of a function $f$ on $\mathbb{R}$ is defined by

$$
\widehat{f}(\omega)=\int_{-\infty}^{\infty} d t f(t) e^{-i \omega t}
$$

\section{A POSITIVITY RESULT}

Let $f$ be a smooth, even and non-negative function on $\mathbb{R}$ which decays rapidly at infinity [including the possibility that $f$ is compactly supported]. Using $f^{1 / 2}$ to denote the pointwise square root of $f$ [i.e., $\left.f^{1 / 2}(t)=\sqrt{f(t)}\right]$, the function $g$ defined by

$$
g(\omega)=\frac{\widehat{f^{1 / 2}}(\omega)}{\sqrt{2 \pi}}
$$

on $\mathbb{R}$ is smooth, real-valued and even, decays rapidly at infinity and obeys

$$
g \star g=\widehat{f},
$$

where the convolution $\star$ is defined by

$$
\left(h_{1} \star h_{2}\right)(\omega)=\int_{-\infty}^{\infty} d \omega^{\prime} h_{1}\left(\omega-\omega^{\prime}\right) h_{2}\left(\omega^{\prime}\right) .
$$

Now let $p$ be a real valued function on $\mathbb{R}^{n}$, growing no faster than polynomially. We use the annihilation and creation operators of the scalar field in $n+1$-dimensional Minkowski 
space to define two families $\left\{\mathcal{O}_{\omega}^{ \pm} \mid \omega \in \mathbb{R}^{+}\right\}$of operators on the Fock space of the Minkowski vacuum by

$$
\mathcal{O}_{\omega}^{ \pm}=\int \frac{d^{n} \mathbf{k}}{(2 \pi)^{n}}\left\{g(\omega-\omega(\mathbf{k})) a(\mathbf{k}) \pm g(\omega+\omega(\mathbf{k})) a^{\dagger}(\mathbf{k})\right\} p(\mathbf{k})
$$

Using the commutation relations and symmetrising the integrand in $\mathbf{k}$ and $\mathbf{k}^{\prime}$, we calculate

$$
\begin{aligned}
\int_{0}^{\infty} d \omega \mathcal{O}_{\omega}^{ \pm \dagger} \mathcal{O}_{\omega}^{ \pm}= & \int_{0}^{\infty} d \omega \int \frac{d^{n} \mathbf{k} d^{n} \mathbf{k}^{\prime}}{(2 \pi)^{2 n}}\left\{g(\omega-\omega(\mathbf{k})) g\left(\omega-\omega\left(\mathbf{k}^{\prime}\right)\right) a^{\dagger}(\mathbf{k}) a\left(\mathbf{k}^{\prime}\right)\right. \\
& +g(\omega+\omega(\mathbf{k})) g\left(\omega+\omega\left(\mathbf{k}^{\prime}\right)\right) a(\mathbf{k}) a^{\dagger}\left(\mathbf{k}^{\prime}\right) \\
& \pm g(\omega-\omega(\mathbf{k})) g\left(\omega+\omega\left(\mathbf{k}^{\prime}\right)\right) a^{\dagger}(\mathbf{k}) a^{\dagger}\left(\mathbf{k}^{\prime}\right) \\
& \left. \pm g(\omega+\omega(\mathbf{k})) g\left(\omega-\omega\left(\mathbf{k}^{\prime}\right)\right) a(\mathbf{k}) a\left(\mathbf{k}^{\prime}\right)\right\} p(\mathbf{k}) p\left(\mathbf{k}^{\prime}\right) \\
= & S^{ \pm}+\int_{0}^{\infty} d \omega \int \frac{d^{n} \mathbf{k}}{(2 \pi)^{n}} g(\omega+\omega(\mathbf{k}))^{2} p(\mathbf{k})^{2}
\end{aligned}
$$

where

$$
\begin{aligned}
S^{ \pm}= & \frac{1}{2} \int \frac{d^{n} \mathbf{k} d^{n} \mathbf{k}^{\prime}}{(2 \pi)^{2 n}}\left\{F\left(\mathbf{k}, \mathbf{k}^{\prime}\right)\left(a^{\dagger}(\mathbf{k}) a\left(\mathbf{k}^{\prime}\right)+a^{\dagger}\left(\mathbf{k}^{\prime}\right) a(\mathbf{k})\right)\right. \\
& \left. \pm G\left(\mathbf{k}, \mathbf{k}^{\prime}\right)\left(a^{\dagger}(\mathbf{k}) a^{\dagger}\left(\mathbf{k}^{\prime}\right)+a\left(\mathbf{k}^{\prime}\right) a(\mathbf{k})\right)\right\} p(\mathbf{k}) p\left(\mathbf{k}^{\prime}\right)
\end{aligned}
$$

and the functions $F$ and $G$ are given by

$$
F\left(\mathbf{k}, \mathbf{k}^{\prime}\right)=\int_{0}^{\infty} d \omega g(\omega-\omega(\mathbf{k})) g\left(\omega-\omega\left(\mathbf{k}^{\prime}\right)\right)+g(\omega+\omega(\mathbf{k})) g\left(\omega+\omega\left(\mathbf{k}^{\prime}\right)\right)
$$

and

$$
G\left(\mathbf{k}, \mathbf{k}^{\prime}\right)=\int_{0}^{\infty} d \omega g(\omega-\omega(\mathbf{k})) g\left(\omega+\omega\left(\mathbf{k}^{\prime}\right)\right)+g(\omega+\omega(\mathbf{k})) g\left(\omega-\omega\left(\mathbf{k}^{\prime}\right)\right) .
$$

The expressions for $F$ and $G$ may be simplified, using the fact that $g$ is even, to obtain

$$
\begin{aligned}
F\left(\mathbf{k}, \mathbf{k}^{\prime}\right) & =\int_{-\infty}^{\infty} d \omega g(\omega-\omega(\mathbf{k})) g\left(\omega-\omega\left(\mathbf{k}^{\prime}\right)\right) \\
& =(g \star g)\left(\omega(\mathbf{k})-\omega\left(\mathbf{k}^{\prime}\right)\right) \\
& =\widehat{f}\left(\omega(\mathbf{k})-\omega\left(\mathbf{k}^{\prime}\right)\right)
\end{aligned}
$$

and, similarly,

$$
G\left(\mathbf{k}, \mathbf{k}^{\prime}\right)=(g \star g)\left(\omega(\mathbf{k})+\omega\left(\mathbf{k}^{\prime}\right)\right)=\widehat{f}\left(\omega(\mathbf{k})+\omega\left(\mathbf{k}^{\prime}\right)\right)
$$

Since the right hand side of Eq. (3.5) is (formally) a manifestly positive operator, we conclude that the expectation value $\left\langle S^{ \pm}\right\rangle_{\psi}$ obeys the following bound

$$
\begin{aligned}
\left\langle S^{ \pm}\right\rangle_{\psi} & \geq-\int_{0}^{\infty} d \omega \int \frac{d^{n} \mathbf{k}}{(2 \pi)^{n}} g(\omega+\omega(\mathbf{k}))^{2} p(\mathbf{k})^{2} \\
& =-\frac{1}{2 \pi} \int_{0}^{\infty} d \omega \int \frac{d^{n} \mathbf{k}}{(2 \pi)^{n}} \widehat{f^{1 / 2}}(\omega+\omega(\mathbf{k}))^{2} p(\mathbf{k})^{2}
\end{aligned}
$$


in all sufficiently well behaved states $\psi$. As we will see, this bound provides the key to our derivation of the quantum inequality (1.7).

We conclude this section with two remarks. Firstly, we have argued rather formally and have interchanged orders of integration at will. Nonetheless, we expect that if $f$ has rapid decay at infinity (e.g., if $f$ is a Schwartz test function) our result can be established rigorously for a dense set of states in the folium of the usual Minkowski vacuum, and conceivably for all Hadamard states on the usual field algebra. Secondly, we do not claim that Eq. (3.11) is the sharpest bound that can be placed on the expectation value of $S^{ \pm}$, and in fact do not expect that the bound is actually attained by any reasonable state $\psi$, as $\psi$ would necessarily belong to the kernel of all $\mathcal{O}_{\omega}^{ \pm}$'s (except, perhaps, for a set of measure zero).

\section{DERIVATION OF THE QUANTUM INEQUALITIES}

The result derived above allows a simple proof of the energy inequalities. With $f$ as in Section [II, define

$$
T_{f}=\int_{-\infty}^{\infty} T_{00}^{\mathrm{ren}}(t, 0) f(t)
$$

so that $\rho_{f, \psi}=\left\langle T_{f}\right\rangle_{\psi}$. By Eq. 2.5) and the fact that $\widehat{f}$ is even, we have

$$
\begin{aligned}
T_{f}= & \frac{1}{2} \int \frac{d^{n} \mathbf{k} d^{n} \mathbf{k}^{\prime}}{(2 \pi)^{2 n}} \frac{\omega(\mathbf{k}) \omega\left(\mathbf{k}^{\prime}\right)+\mathbf{k} \cdot \mathbf{k}^{\prime}}{2\left(\omega(\mathbf{k}) \omega\left(\mathbf{k}^{\prime}\right)\right)^{1 / 2}}\left\{F\left(\mathbf{k}, \mathbf{k}^{\prime}\right)\left(a^{\dagger}(\mathbf{k}) a\left(\mathbf{k}^{\prime}\right)+a^{\dagger}\left(\mathbf{k}^{\prime}\right) a(\mathbf{k})\right)\right. \\
& \left.+G\left(\mathbf{k}, \mathbf{k}^{\prime}\right)\left(a^{\dagger}(\mathbf{k}) a^{\dagger}\left(\mathbf{k}^{\prime}\right)+a\left(\mathbf{k}^{\prime}\right) a(\mathbf{k})\right)\right\} \\
& +\frac{1}{2} \int \frac{d^{n} \mathbf{k} d^{n} \mathbf{k}^{\prime}}{(2 \pi)^{2 n}} \frac{m^{2}}{2\left(\omega(\mathbf{k}) \omega\left(\mathbf{k}^{\prime}\right)\right)^{1 / 2}}\left\{F\left(\mathbf{k}, \mathbf{k}^{\prime}\right)\left(a^{\dagger}(\mathbf{k}) a\left(\mathbf{k}^{\prime}\right)+a^{\dagger}\left(\mathbf{k}^{\prime}\right) a(\mathbf{k})\right)\right. \\
& \left.-G\left(\mathbf{k}, \mathbf{k}^{\prime}\right)\left(a^{\dagger}(\mathbf{k}) a^{\dagger}\left(\mathbf{k}^{\prime}\right)+a\left(\mathbf{k}^{\prime}\right) a(\mathbf{k})\right)\right\}
\end{aligned}
$$

with $F$ and $G$ given by Eqs. (3.9) and (3.10). Clearly, $T_{f}$ is a finite sum of operators of the form $S^{ \pm}$. We therefore apply the bound (3.11) for the various cases

$$
p(\mathbf{k})=\frac{\omega(\mathbf{k})}{\sqrt{2 \omega(\mathbf{k})}}, \quad p(\mathbf{k})=\frac{k_{i}}{\sqrt{2 \omega(\mathbf{k})}}, \quad p(\mathbf{k})=\frac{m}{\sqrt{2 \omega(\mathbf{k})}}
$$

and add the results, obtaining

$$
\begin{aligned}
\rho_{f, \psi} & \geq-\frac{1}{2 \pi} \int_{0}^{\infty} d \omega \int \frac{d^{n} \mathbf{k}}{(2 \pi)^{n}} \omega(\mathbf{k}) \widehat{f^{1 / 2}}(\omega+\omega(\mathbf{k}))^{2} \\
& =-\frac{C_{n}}{2 \pi} \int_{0}^{\infty} d \omega \int_{m}^{\infty} d \omega^{\prime} \widehat{f^{1 / 2}}\left(\omega+\omega^{\prime}\right)^{2} \omega^{\prime 2}\left(\omega^{\prime 2}-m^{2}\right)^{n / 2-1}
\end{aligned}
$$

for all (sufficiently well-behaved) states $\psi$. Here, $C_{n}$ is equal to the area of the unit $n-1$ sphere divided by $(2 \pi)^{n}$ [with the convention that $\left.C_{1}=1 / \pi\right]$, that is,

$$
C_{n}=\frac{1}{2^{n-1} \pi^{n / 2} \Gamma\left(\frac{1}{2} n\right)}
$$


If we now make the change of variables

$$
u=\omega+\omega^{\prime} \quad v=\omega^{\prime}
$$

we find

$$
\begin{aligned}
\rho_{f, \psi} & \geq-\frac{C_{n}}{2 \pi} \int_{m}^{\infty} d u\left(\widehat{f^{1 / 2}}(u)\right)^{2} \int_{m}^{u} d v v^{2}\left(v^{2}-m^{2}\right)^{n / 2-1} \\
& =-\frac{C_{n}}{2 \pi(n+1)} \int_{m}^{\infty} d u\left(\widehat{f^{1 / 2}}(u)\right)^{2} u^{n+1} Q_{n}\left(\frac{u}{m}\right)
\end{aligned}
$$

where the functions $Q_{n}(x)$ are defined by Eq. (1.8), thus completing the derivation of our general quantum inequality Eq. (1.7). We note that each $Q_{n}(x)$ is a positive function with $Q_{n}(1)=0$ and $Q_{n}(x) \rightarrow 1$ as $x \rightarrow \infty$. For $n \geq 2, Q_{n}(x)$ is strictly increasing on $\mathbb{R}^{+}$, while $Q_{1}(x)$ exhibits a maximum near $x=1.8$ and decreases thereafter - see Figures 11 and 2 .

To conclude this section, we consider the scaling behaviour of (1.7). Let $f_{\lambda}(t)$ be the scaled function

$$
f_{\lambda}(t)=\lambda^{-1} f(t / \lambda)
$$

This function has the same integral over $\mathbb{R}$ as $f$ but with $\lambda$ times the characteristic width. It is easy to see that

$$
\left(\widehat{f_{\lambda}^{1 / 2}}(u)\right)^{2}=\lambda\left(\widehat{f^{1 / 2}}(u)\right)^{2}
$$

from which it follows that the bound for sampling function $f_{\lambda}$ at mass $m$ is equal to $\lambda^{-(n+1)}$ times the bound for sampling function $f$ at mass $\lambda m$. This is the expected scaling behaviour and is also exhibited by the quantum inequalities (1.3) and (1.4) of Ford and Roman. Furthermore, it is clear that the value of the bound (1.7) tends to zero for each fixed $f$ as $m \rightarrow \infty$. Thus we have

$$
\lim _{\lambda \rightarrow \infty} \lambda \rho_{f_{\lambda}, \psi} \geq 0
$$

and we recover the averaged weak energy condition in the limit (cf. [12]).

\section{SPECIAL CASES}

In this section, we briefly discuss the most interesting cases of the general quantum inequality derived above, and compare our results with those of Flanagan [11] and Ford and Roman [3].

\section{A. Two dimensions}

As mentioned in the introduction, Flanagan has derived an apparently optimal quantum inequality for massless 2-dimensional field theory [11]. Substituting $n=1$ and $m=0$ into our bound Eq. (1.7) and using the fact that $Q_{1}(x) \rightarrow 1$ as $x \rightarrow \infty$, we find 


$$
\rho_{f, \psi} \geq-\frac{1}{4 \pi^{2}} \int_{0}^{\infty} d u u^{2}\left(\widehat{f^{1 / 2}}(u)\right)^{2} .
$$

The integrand is an even function in $u$, so we may extend the range of integration to the whole of $\mathbb{R}$ and then employ Parseval's theorem to yield a $t$-space version of the quantum inequality

$$
\rho_{f, \psi} \geq-\frac{1}{4 \pi} \int_{-\infty}^{\infty} d t\left(f^{1 / 2^{\prime}}(t)\right)^{2}=-\frac{1}{16 \pi} \int_{\operatorname{supp} f} d t \frac{\left(f^{\prime}(t)\right)^{2}}{f(t)}
$$

which should be compared with Flanagan's bound (1.5). Our bound is seen to be weaker by a factor of $\frac{3}{2}$; there is no contradiction because we do not expect our bound to be optimal. Since Flanagan's bound is six times stronger than that of Ford and Roman when applied to the Lorentzian sampling function, our bound is accordingly four times stronger in this case.

In the 2-dimensional massive case, the quantum inequality (1.7) becomes

$$
\rho_{f, \psi} \geq-\frac{1}{4 \pi^{2}} \int_{0}^{\infty} d u u^{2}\left(\widehat{f^{1 / 2}}(u)\right)^{2} Q_{1}\left(\frac{u}{m}\right)
$$

with $Q_{1}$ given by

$$
Q_{1}(x)=\left(1-x^{-2}\right)^{1 / 2}+x^{-2} \log \left(x+\left(x^{2}-1\right)^{1 / 2}\right) .
$$

Figure 1 shows that $Q_{1}(x)$ exhibits a maximum value of approximately 1.2 near $x=1.8$. We cannot exclude the possibility that massive fields in 2-dimensions can exhibit slightly stronger negative energy densities than massless fields can (by a factor of at most 1.2). Interestingly, a similar phenomenon occurs in Ford and Roman's treatment [3] so it would be worthwhile to determine whether this is indeed so, or whether the peak is an artifact of the argument (as Ford and Roman suggest).

\section{B. Four dimensions}

Just as in the 2-dimensional case discussed above, the 4-dimensional quantum inequality takes a particularly simple form for massless fields:

$$
\rho_{f, \psi} \geq-\frac{1}{16 \pi^{3}} \int_{0}^{\infty} d u\left(\widehat{f^{1 / 2}}(u)\right)^{2} u^{4}=-\frac{1}{16 \pi^{2}} \int_{-\infty}^{\infty} d t\left(f^{1 / 2^{\prime \prime}}(t)\right)^{2} .
$$

In particular, for the Lorentzian function we have

$$
\rho_{L, \psi} \geq-\frac{1}{16 \pi^{2}} \int_{-\infty}^{\infty} d t \frac{\left(2 t^{2}-\tau^{2}\right)^{2} \tau}{\pi\left(t^{2}+\tau^{2}\right)^{5}}=-\frac{27}{2048 \pi^{2} \tau^{4}}
$$

which is $\frac{9}{64}$ of Ford and Roman's result (1.3) in this case [recall that $G(0)=1$ ]. This entails a slight tightening of the constraints on traversable wormholes [8].

Finally, we state the form of Eq. (1.7) in the 4-dimensional massive case. In terms of the Fourier transform of $f$, we have 


$$
\rho_{f, \psi} \geq-\frac{1}{16 \pi^{3}} \int_{m}^{\infty} d u\left(\widehat{f^{1 / 2}}(u)\right)^{2} u^{4} Q_{3}\left(\frac{u}{m}\right)
$$

where

$$
Q_{3}(x)=\left(1-x^{-2}\right)^{1 / 2}\left(1-\frac{1}{2 x^{2}}\right)-\frac{1}{2 x^{4}} \log \left(x+\left(x^{2}-1\right)^{1 / 2}\right) .
$$

As Figure 2 makes clear, the function $Q_{3}$ is bounded between zero and unity. Accordingly the bound (5.5) is also a lower bound for massive fields: in 4 dimensions [indeed, in any spacetime dimension greater than or equal to three] the effect of introducing a mass cannot decrease the averaged energy density below the massless bound.

\section{CONCLUSION}

We have given a simple derivation of a quantum inequality for the free real scalar field in Minkowski space of any dimension, which allows more general sampling functions than previously possible. In particular, our derivation allows for compactly supported sampling functions and therefore removes any remaining doubt that the quantum inequalities of Ford and Roman might rely on subtle large scale effects to cancel local negative energy densities. In conclusion, we make various remarks.

First, the derivation given here has been somewhat formal and lacking in mathematical rigour. However, we hope that our argument is simple enough that a rigorous formulation might be established without too much difficulty, and intend to return to this issue elsewhere. Two elements of our discussion need to be more precisely specified: namely the class of sampling functions and the class of quantum states for which Eq. (1.7) is valid. We have required that the sampling function $f$ be even primarily for convenience, and expect that this condition may be removed. In addition, we may speculate as to whether it is necessary that $f$ be smooth. Of course, a necessary condition on $f$ is is that the integral on the right hand side of Eq. (1.7) should converge; this amounts to a smoothness condition on $f$ which becomes more stringent as the spacetime dimension increases (and which is always satisfied if $f$ is actually smooth). Thus, it may be that the smoothness of $f$ could be relaxed to $C^{k}$ where $k$ depends on $n$. However, it is clearly important that $f$ has some degree of continuity. As an example, suppose $f$ is the characteristic function for the interval $[-\tau, \tau] \subset \mathbb{R}$. We have

$$
\left(\widehat{f^{1 / 2}}(\omega)\right)^{2}=\frac{4 \sin ^{2} \omega \tau}{\omega^{2}}
$$

from which it follows that the integral in Eq. (1.7) diverges for any $n \geq 1$. Thus the quantum inequality provides no information in this case, which is consistent with results of Garfinkle (quoted by Yurtsever in [13] - see particularly footnote [1] therein) that the integral of $T_{00}^{\text {ren }}$ over sharply defined boxes in spacetime can be unboundedly negative. As mentioned above, the class of quantum states for which Eq. (1.7) holds must also be clarified. It is likely that quantum inequalities will hold for a dense class of states in the Fock space built on the Minkowski vacuum; more generally, we hope that such inequalities might be established for the class of (globally) Hadamard states [14, 15. 
Second, we have seen that our bound is weaker by a factor of $\frac{3}{2}$ than the optimal bound proposed by Flanagan [11], which was derived using special features of 2-dimensional massless field theory. It would be interesting to investigate whether our argument could be improved to replicate Flanagan's result and perhaps to obtain optimal bounds for the massive case and also higher dimensional spacetimes.

Finally, we anticipate no particular difficulties in generalising our argument to provide quantum inequalities in curved spacetime. Again, we intend to return to this elsewhere. 


\section{REFERENCES}

[1] H. Epstein, V. Glaser and A. Jaffe, Nuovo Cim. 361016 (1965)

[2] L.H. Ford, Phys. Rev. D43 3972 (1991)

[3] L.H. Ford and T.A. Roman, Phys. Rev. D55 2082 (1997)

[4] M.J. Pfenning and L.H. Ford, Phys. Rev. D55 4813 (1997)

[5] M.J. Pfenning and L.H. Ford, Phys. Rev. D57 3489 (1998)

[6] L.H. Ford, M.J. Pfenning and T.A. Roman, Phys. Rev. D57 4839 (1998)

[7] A.D. Helfer, Class. Quantum Grav. 13 L129 (1996)

[8] L.H. Ford and T.A. Roman, Phys. Rev. D53 5496 (1995)

[9] M.J. Pfenning and L.H. Ford, Class. Quantum Grav. 141743 (1997)

[10] L.H. Ford and T.A. Roman, Phys. Rev. D53 1988 (1996)

[11] E.E. Flanagan, Phys. Rev. D56 4922 (1997)

[12] L.H. Ford and T.A. Roman, Phys. Rev. D51 4277 (1995)

[13] U. Yurtsever, Phys. Rev. D51 5797 (1995)

[14] B.S. Kay and R.M. Wald, Phys. Rep. 20749 (1991)

[15] M.J. Radzikowski, Commun. Math. Phys. 179529 (1996) 


\section{FIGURES}

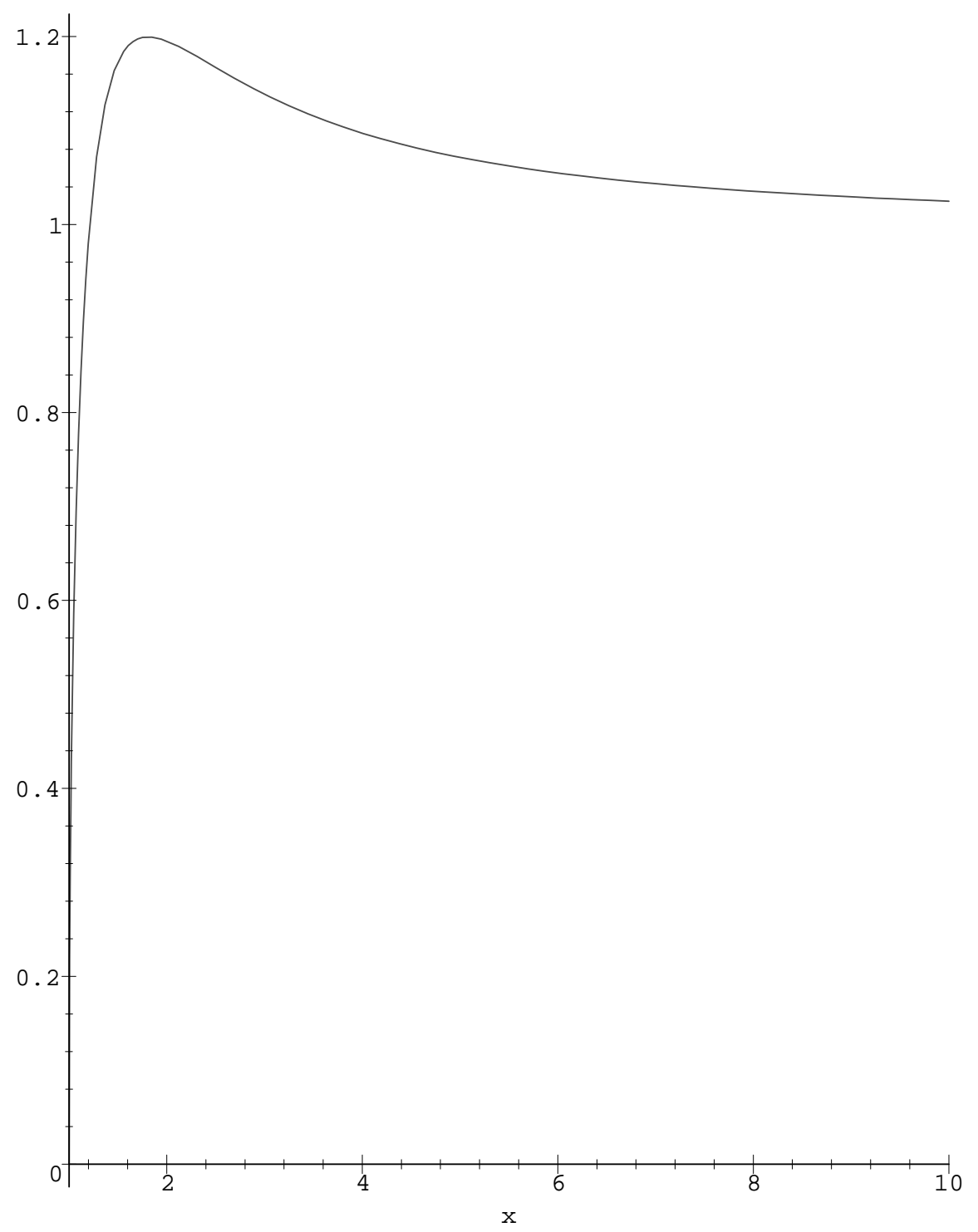

FIG. 1. Graph of $Q_{1}(x)$. 


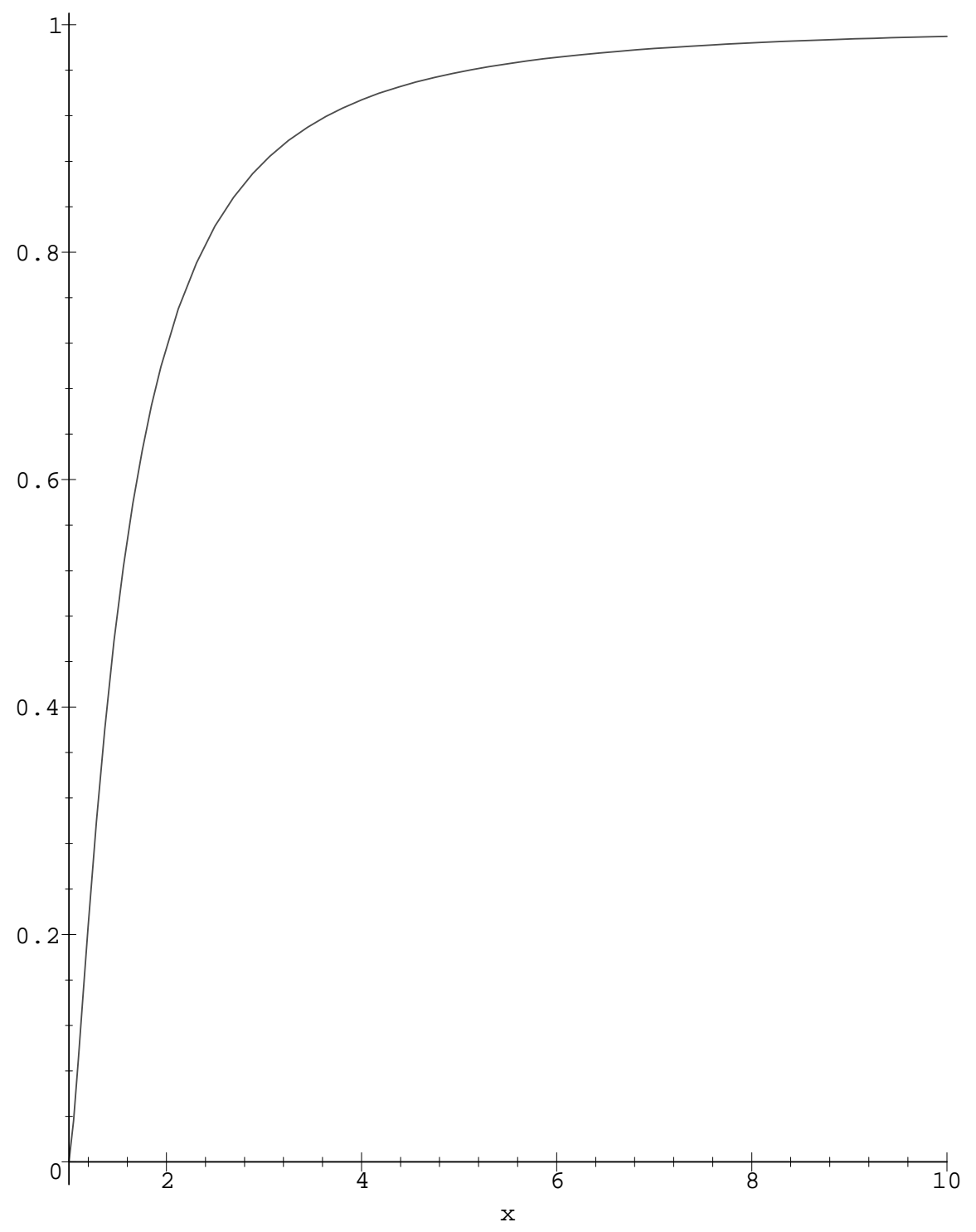

FIG. 2. Graph of $Q_{3}(x)$. 